\title{
Data acquisition time minimization in FANET-based IoT networks
}

\author{
Alaa Taima Albu-Salih ${ }^{1, *}$, Hayder Ayad Khudhair $^{2}$, Osama Majeed Hilal ${ }^{1}$ \\ ${ }^{l}$ Ministry of Education, General Directorate for Education in Al-Qadisiyah, Iraq \\ ${ }^{2}$ Ministry of Education, General Directorate for Education in Al-Najaf Al-Ashraf, Iraq \\ *Corresponding author: alaa.taima@qu.edu.iq
}

\begin{abstract}
Unmanned aerial vehicles (UAVs) is widely used in many military, and civilian applications. UAVs communicate in a Flying Ad hoc Network (FANET) environment where UAVs communicate with each other through an ad hoc network without infrastructure. FANET provide a flexible platform for internet of things (IoT) applications by playing different roles in IoT such as mobile data collector. In fact, in deadline based IoT applications, the deadline is restricted to the critical application level. And as a result, this deadline for data acquisition is not adequate, and FANET cannot collect data from the sensed area with the predetermined deadline. In this paper, a novel efficient data gathering approach for IoT using FANET is proposed. The main objective of this approach is to solve the problem of insufficient deadlines given by FANET in IoT-based deadline applications. Authors will first provide a multi-objective optimization model as a MILP optimization model to solve this problem, and then normalize and add two weighing coefficients to solve the MILP model. The results obtained in the simulation show that the proposed approach can provide efficient data acquisition while guaranteeing the deadline time.
\end{abstract}

Keywords: Data acquisition, deadline, IoT, FANET, UAV.

\section{Introduction}

In the last few years, unmanned aerial vehicles (UAVs), commonly known as drones (Motlagh et al.,2016), is an emerging technology that has spread widely in different civil and military applications (Otto et al,2018; Shakhatreh et al.,2019), like border surveillance, disaster management, transport of medical supplies, traffic monitoring, battlefield surveillance, border surveillance, forestry fire monitoring, animal tracking, remote sensing and smart cities (Al-Turjman \& Zahmatkesh,2020). The use of a group of small UAVs, instead of one large UAV, exposes several advantages in terms of performance, adaptability, agility and easy deployment. Using several UAVs has helped to design a new type of snetwork called Flying Ad Hoc Network (FANET) (Bekmezci et al.,2013; Sharma et al., 2017).

FANET nodes (UAV) are used to collect data from the Internet of Things (IoT) as mobile collectors (Liu et al., 2020). Each UAV in FANET network may play an important role in IoT, consisting of devices with a small battery. These devices typically cannot be spread over long distances, due to the IoT devices' energy limitation (Albu-Salih \& Seno, 2018). Thus, UAVs can then travel dynamically to IoT devices, collect data and send it to other UAVs for further processing beyond the IoT device communication ranges (Mozaffari et al.,2017), as seen in Figure 1.

Some applications that are real-time, soft, or hard need to collect or send data at a specific deadline time. Therefore, a data acquisition deadline time should be defined as to the type of application (Dasgupta \& Yoon, 2017). In many deadline-based IoT applications, the data acquisition time is determined by priority 
and the critical level of the data previously collected. Also, In In many deadline-based IoT applications, the remote user can request different data acquisition deadlines (Albu-Salih \& Seno, 2018).

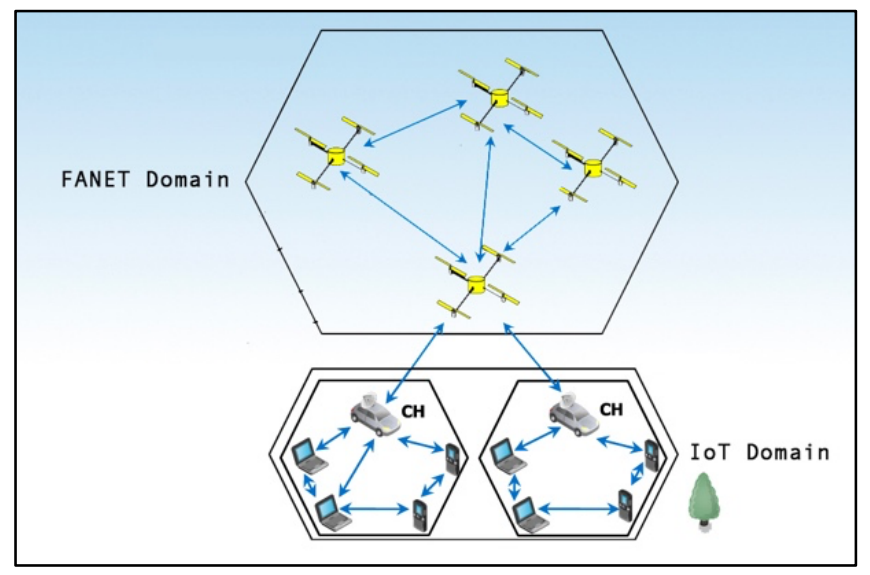

Fig. 1. FANET-Aided IoT Networks.

Forest surveillance, pollution monitoring, battlefield surveillance animal tracking and environment monitoring are examples of deadline based IoT applications (Boursianis et al., 2020). In a forest surveillance application, wireless sensor nodes are mounted for fire and smoke recognition in the forest. UAV periodically flies above the targeted area to collect data at specified points from wireless sensor nodes. Such data collection should be dependent on the specified deadline (Khan et al., 2014). Another example is the use of UAVs in deadline-based IoT applications, including monitoring of hazard assessments for a given area. Therefore, a constructive relationship can be formed between a UAV and a smart city's security force to monitor strategic sites (Tuyishimire et al., 2017).

In fact, in deadline based IoT applications, the deadline is restricted to the critical application level, and as a result, this deadline for data acquisition is not sufficient, and data cannot be collected by FANET from the sensed area with predetermined deadline. The main goal of this paper is to minimize the time needed for data collection within a predetermined period time, which should be efficiently performed in terms of UAV and IoT energy consumption. The problem of inadequate deadline time is mathematically formulated as mixed-integer linear (MILP) optimization model.

The contributions of this paper are:

1) Providing an efficient data gathering approach using the number of UAVs in FANET-aided IoT networks, by optimizing the travelled time of UAVs.

2) The problem of inadequate deadline time is mathematically formulated as a MILP optimization model.

3) Normalization and coefficients are added to solve this model.

4) The efficiency of the proposed approach is compared to that of a greedy approach in terms of UAV travel time.

The rest of this paper is arranged as follows. The related work and associated problems in FANET networks are discussed in Section 2. In section 3, the proposed approach stages are presented. In section 4, the problem definition and formulation are presented. The authors evaluate the performance of the proposed model and compare it with the greedy method in Section 5. In Section 6, the results and suggestions for future work are summarized. 


\section{Related works}

Despite practical FANET applications in IoT networks, many technical problems, such as deadline-based data gathering, should be examined. Although several works are designed for UAV-assisted data gathering they are not necessarily suited to FANET deadline-based data gathering. Several works with different techniques are proposed for FANET trying to provide efficient data gathering. Therefore, authors first review the relevant FANET-based data gathering schemes according to the problem, and then examine each of these works and describe the advantages and drawbacks of each work.

The authors in (Caillouet et al., 2019) discuss the Aerial Data Collection Problem (ADCP) by using a swarm of flying devices from multiple mobile WSN is located on the ground. They proposed a framework for the assessment and comparison of algorithms for wireless flying drones for data collection and monitoring strategies. The main objective of this framework is to decrease the overall cost of deployment.

In (Alfattani et al., 2019), a framework for wireless sensor networks based on multiple UAVs. The goal of this framework is to minimize deployment and operational costs concerning budget and power constraints.

(Okcu \& Soyturk, 2014) examines the problem of coverage where UAV is used as a mobile sink. To solve the previous problem, an RSSI-based clustering in WSNs using UAVs as mobile sinks is proposed. The goal of this strategy is to reduce the consumption of energy while avoiding unnecessary cluster head formations and avoiding uncovered network nodes.

Gong et al. (2018) investigated the problem of flight time minimization for a UAV that used to gather data from a group of WSN nodes. The goal is to decrease the overall flight time of UAVs from a starting to a destination point by optimizing the speed of the UAV, the division of intervals and the transmitting power of the sensors.

A multi-UAV assisted WSN design is considered by Zhan \& Zeng (2019), where UAVs travel to gather data from wireless sensor nodes. By optimizing the trajectory of UAVs and the WSN association, the aim is to reduce the overall mission completion time for all UAVs. To solve the above problem, they suggested two schemes (Hmode and Fmode). When each UAV only collects data while hovering, the hovering mode (Hmode) scheme is used. The Flying Mode (Fmode) system is used only when each UAV gathers information while flying.

Dong et al. (2014) designed a novel data gathering algorithm, employing both the UAV and mobile agents (MAs) to separately collect and process data in WSN. The aim of this algorithm to provide time and energy-efficient for any density of the network.

The problem of gathering data from a WSN with UAV controlled altitude is discussed in (Ali et al., 2019). This algorithm is evaluated at a constant speed, so that when a UAV arrives in the relay node range, data transmission is initiated.

Goudarzi et al. (2019) developed a novel approach to path planning that uses a smooth and short data collection path for UAV motion. This approach aims to efficiently collect data with a low energy consumption and high packet delivery rate. The movement of UAVs is managed locally onboard each UAV with the Lyapunov function, and redundant data on the sensors are removed using the Kalman filter method.

The UAV-assisted WSN system, where a UAV is deployed as an auxiliary data collection node for a delay-tolerant sensor, was investigated by Liu et al. (2019). The goal is to decrease the energy consumption of transmission while guaranteeing completion of the transmission. Both the transmission strategy of the sensor node and the problem of UAV trajectory optimization are discussed in this paper. 
To collect data from wide-area WSN, Ho et al. (2015) used a UAV as a data mule to then relay this data to a base station at its convenience. The goal of this algorithm is to find the optimal WSN communication topology and UAV path while taking bit error rate of all WSN nodes, energy consumption and flight time of the UAV into account.

In a monitoring scenario when considering the quality of information collection, Qin et al. (2019) discussed the minimization of the mission completion time problem for a multi-UAV network. This problem has been formulated as a nonconvex mixed-integer model. They have proposed FHF trajectory planning algorithm to optimize this model. Deadline time has not been studied in the previous works for UAV-based data gathering schemes.

\section{The proposed framework}

To address the problem of an insufficient deadline time in FANET-aided IoT networks, a novel efficient data gathering approach for FANET was proposed. As discussed earlier, the main problem of this paper is to minimize UAV's travel time to collect data from ground IoTs within a predetermined deadline, which should be efficiently performed in terms of FANET and IoT's energy consumption.

According to the assumptions of the network, the stages needed to implement the proposed approach to minimize travel time of the UAV are:

\subsection{Clustering}

First, authors cluster the nodes into distributed manner to decide where the UAVs are to be located to collect data from the IoT nodes. In order to effectively cluster IoTs for data transmission to the network in terms of energy consumption, a preliminary clustering would perform so that the average distance of a cluster members reduced and the IoT requires the least amount of energy required to transfer data to the head of the cluster. To do clustering, the threshold for sending IoT nodes determined at the network boundary, taking the energy threshold parameter into account, and at this distance, each node determines its neighbours. Based on the network density, the threshold is the number of nodes in every cluster and whose neighbours are more than or equal to this threshold. In this case, the IoTs specify the number of clusters needed to send effective data, and these cluster centres are regarded as the location of drone deployment. The data is transmitted from the IoT nodes to the UAV after the cluster has been established and the UAV is deployed at the centre of each cluster, and the UAV receives data from the cluster heads during the deadline time.

\subsection{Virtual nodes determination}

At this stage, authors are trying to solve the main paper problem after completing the clustering phase and deciding the meeting points for the UAV. In this stage, authors presume that the IoT nodes in the network are stationary. This is the form to decide the effective path in the network: The meeting points of the UAV are used as network nodes, taking into account the centre of the clusters selected in the previous stage. Furthermore, as described in the main form of the problem, one of the problem-solving objectives is to determine the optimum number of UAVs for network coverage, which means that the problem solution should propose several paths for moving UAVs at these nodes, which simultaneously scroll through meeting points and collect data. To determine the number of these routes and to determine the number of 
UAVs present on the network, the virtual nodes 0 and $n+1$ are considered to be the beginning and end nodes of all UAVs' routes. Therefore, by defining nodes 1 through $\mathrm{n}$ as cluster centres and virtual nodes. Authors define $N$ set as a network node as follows:

$$
N=\{0,1,2,3,4, \ldots \ldots ., n, n+1\}
$$

Assuming a directed graph $(G)$ for performing the path problem as follows:

$$
G=(N, E)
$$

E denotes the travel distance between the nodes. In this way, the problem is raised as follows: Finding the shortest $u$ path from $(0)$ to $(n+1)$ node with the following states: A path must be less time than the specific deadline time. Except for source and destination nodes, the paths do not share the edges and nodes. The path should be as short as possible. Of course, the number of paths $(u)$ that are actually the same number of UAVs must be the possible minimum number.

\subsection{UAV traveled time minimization}

The network manager had determined the deadline for the processing of network data in previous stages. The goal of this stage is to spend the minimum amount of time necessary for gathering data from the IoT nodes. The minimum amount of time required for network data to be collected and the minimum number of UAVs required for data collection are two of the goals of this paper. These two targets are mutually related such that the data acquisition time is decreased by increasing the number of UAVs to a maximum number of clusters but not by maximizing the number of UAVs. Thus, this stage aims to suggest a model that achieves a reasonable balance between these two goals by looking at this correlation, assigning weights to the dual goals and comparing various states.

\section{Problem formulation and solution}

In this section, the authors first formulate the inadequate deadline time problem as a mathematical optimization model, and then we solve the proposed model.

\subsection{Problem formulation}

As discussed in previous sections, to solve the main problem of the paper, first, in accordance with the initial stage of the proposed method, and to minimize the UAV travelled time, efficient clustering was performed and then, by forming the network graph and according to the description of the second stage of the proposed approach, the main problem of the paper in the context of the IoT node scenario is solved by MILP model, which is explained as follow:

It can be clarified that $U$ (the maximum number of UAVs) is determined in such a way that the total length of the route to be collected for gathering data from the network is calculated, and by calculating the time required to traverse this route at the speed of the UAV, and comparing this time to the deadline time. $U$ is calculated as follows:

$$
U=\frac{(((\operatorname{Max}(E)+\operatorname{Min}(E)) / 2 *(n-1)) / v)}{\tau}
$$

Definitions of symbols and variables of the problem is used in MLIP model are explained in Table 1. 
Table 1. Problem Parameters and Variables

\begin{tabular}{|c|c|}
\hline Symbol & Definition \\
\hline$U$ & Max number of UAVs \\
\hline$N$ & IoT network nodes \\
\hline $\mathrm{d}_{\mathrm{k}}$ & Max distance each UAV travelled \\
\hline $\mathrm{d}_{\mathrm{i}, \mathrm{j}}$ & Euclidean distance from node $\mathrm{i}$ to $\mathrm{j}$ \\
\hline $\mathrm{v}$ & UAV Movement speed \\
\hline$\tau$ & The deadline time of data collection \\
\hline $\mathbf{n}$ & Number of clusters \\
\hline $\mathbf{t}$ & Time required to receive data by UAV per cluster \\
\hline $\mathbf{x}_{\mathbf{i}, \mathbf{j}}^{\mathbf{k}}$ & The movement of the UAV with the index k from cluster head i to from cluster head $\mathrm{j}$ \\
\hline$u$ & \\
\hline $\mathrm{y}_{\mathbf{i}}$ & Number of needed UAVs \\
\hline
\end{tabular}

The objective function of this paper can be shown as follows, which is a MILP problem:

$$
\min \sum_{k \in U} \sum_{i \epsilon N \backslash\{n+1\}} \sum_{j \in N \backslash\{0\}} d_{i j} x_{i j}^{k}
$$

$\min \sum_{k \in U} T_{k}$

Objective functions aim is to minimize the energy of UAVs to move between cluster heads (function 2), and the minimum required time to collect data from IoT nodes (function 3 ).

The objective function has many constraints that can be classified into classes (Arrival UAV at nodes, Sub-tour elimination Constraint, UAV maximum flight distance and minimum travel time of UAV). Constraints include:

$$
\begin{aligned}
& \sum_{k \in U} \sum_{\in N \backslash\{0\}} x_{i j}^{k}=1 \quad \forall i \in\{1, n\} \\
& \sum_{k \in U} \sum_{j \in\{0, \ldots, n\}} x_{j i}^{k}=1 \quad \forall i \in\{1, n\} \\
& \sum_{k \in U} \sum_{i \in N \backslash\{0\}} x_{0 j}^{k}=u \\
& \sum_{\mathrm{k} \in \mathrm{U}} \sum_{\mathrm{j} \in\{0, \ldots, \mathrm{n}\}} \mathrm{x}_{\mathrm{j}, \mathrm{n}+1}^{\mathrm{k}}=u . \\
& \sum_{k \in U} \sum_{i \in\{0, \ldots, n\}} x_{i 0}^{k}=0 \\
& \sum_{k \in U} \sum_{\in N \backslash\{0\}} x_{n+1 j}^{k}=0 \\
& \forall p \in \mathrm{N} \backslash\{0, \mathrm{n}+1\}, \forall k \in U \\
& x_{i i}^{k}=0, \forall i \forall k \\
& \forall i, j \in N \backslash\{0, n+1\}, i \neq j \\
& \sum_{i \in N\{n+1\}} \sum_{j \in N\{0\}} X_{i j}^{k} d_{i j} \leq d_{k}^{k}-\sum_{j \in N\{n+1\}} x_{p j}^{k}=0 \\
& \sum_{i \in N \backslash\{n+1\}} \sum_{j \in \mathrm{N}\{0\}} x_{i j}^{k}\left(\frac{d_{i j}}{v}+t_{i}\right) \leq T_{k} \\
& \forall k \in U
\end{aligned}
$$




$$
T_{k} \leq \tau
$$

Every node in the middle is only linked to the output node in Constraint (4). In Constraint (5), any middle node is only to be associated with the input node. Constraint (6) ensures the number of node 0 outputs equals $u$. Constraint (7) guarantees that the number of node $n+1$ inputs equal $u$. Constraints (8) ensure that the number of node 0 's inputs equals 0 . In Constraint (9), the number of node $n+1$ outputs is equals 0 . In Constraint (10), input flow is equal to output flow at each middle node. Constraint (11) ensures that there is no loop in the node. Constraint (12) ensures that the route does not have a round. The maximum distance covered by any UAV is predetermined in Constraint (13), and that the maximum distance covered must not exceed the maximum distance covered by any UAV. In Constraint (14), the time traveled on each route should not exceed the UAV's maximum travelled time. In Constraint (15), maximum movement time $\left(T_{k}\right)$ of $U A V_{K}$ must not surpass a deadline time $(\tau)$.

\subsection{Proposed Solution}

The goal of the proposed model is a multi-objective function, as described in the presented model, which is considered as follows to solve the proposed model:

$\min \left(\sum_{k \in U} \sum_{i \in N \backslash\{n+1\}} \sum_{j \in N \backslash\{0\}} d_{i j} x_{i j}^{k}+\sum_{k \in U} T_{k}\right)$

The objective function of this paper is in a multi-objective form, depending on the proposed model. Authors apply the following two steps before authors solve the proposed model:

a) Objective Normalization: Two parts of the objective functions are effectively normalized by measuring the total paths travelled by UAV at the network level to get them close to the numerical value constraints:

$\max _{\text {total }_{\text {paths }}}=((\operatorname{Max}(E)+\operatorname{Min}(E)) / 2) *(N-U)$

When $n$ nodes are reached by a single UAV, the length of the path is an edge of $n-1$ and the $n-1$ edge with UAV reaches all $n$ nodes, the $U-1$ edge is dropped. Therefore, the $N-U$ edge remains, which $U$ drones can simultaneously travel around.

The first part of the objective function is normalized by taking into account the optimum travel value of the UAV in the objective ratio to this max value and the resulting number is within $[0,1]$. For normalization in the second part, the UAV's max travel time is divided by the deadline time $(\tau)$.

$\min \left(\left(\frac{\sum_{k \in U} \sum_{i \epsilon N \backslash\{n+1\}} \sum_{j \epsilon N \backslash\{0\}} d_{i j} \cdot x_{i j}^{k}}{\text { max_total_paths }_{-}}\right)+\left(\frac{\sum_{k \in U} T_{k}}{\tau}\right)\right)$

b) Weighting Coefficients: The coefficients $\alpha$ and $\beta$ are specified and valued as significant weights of each part of the objective in order to weigh each part of the objective function.

Based on the above discussions, the objective of the proposed model is considered as follows:

$\min \left(\alpha\left(\frac{\sum_{k \in U} \sum_{i \epsilon N \backslash\{n+1\}} \sum_{\epsilon N \backslash\{0\}} d_{i j} x_{i j}^{k}}{\text { max_total_paths }}\right)+\beta\left(\frac{\sum_{k \in U} T_{k}}{\tau}\right)\right)$

The multi-objective function is minimized by taking the minimum travel time and travelled distance of UAV into account. Two objectives are considered by normalizing them, applying two weighting coefficients $\alpha$ and $\beta$ to them, and giving them a priority. The number of UAVs, for instance, increases when 
energy is a priority. If the priority is the total UAV travel time, energy will reach a fair value but the total UAV travel time will not be released from the constraints.

Table 2. The effect of choosing alpha and beta on the proposed model solution $N=10$

\begin{tabular}{|c|c|c|c|c|c|c|c|c|}
\hline $\boldsymbol{N}$ & $\boldsymbol{\tau}$ & $\boldsymbol{\alpha}$ & $\boldsymbol{\beta}$ & $\boldsymbol{U}$ & $\boldsymbol{u}$ & $\boldsymbol{u}_{\boldsymbol{c h}}$ & $\boldsymbol{u}_{\boldsymbol{t r j}}$ & Traveled Time \\
\hline \multirow{3}{*}{10} & \multirow{2}{*}{50} & 0.1 & 0.9 & 6 & 4 & 1 & 3 & 105 \\
\cline { 2 - 9 } & \multirow{2}{*}{70} & 0.9 & 0.1 & 6 & 6 & 4 & 2 & 26 \\
\cline { 2 - 8 } & & 0.1 & 0.9 & 7 & 4 & 1 & 3 & 107 \\
\hline
\end{tabular}

Table 2 shows the effect of choosing the $\alpha$ and $\beta$ coefficients for the proposed model solution with and different deadline values in $N=10$ and different deadline times $(\tau=50$ and 70$)$. This table shows that the current paper aims to reduce the time travelled by assigning UAVs to each cluster in situations where the coefficient of the minimum energy objective is the dominant value, by considering $u_{c h}$ and $u_{t r j}$, which are the number of UAVs moving in the network and the number of UAVs assigned only to a cluster, respectively. However, the number of UAVs allocated to each cluster decreases in conditions in which the minimum UAV objective coefficient is dominant. Therefore, UAVs aim to cover all clusters and collect data along the travel route. When the coefficient of the least UAV movement time is dominant, the UAV travel time will decrease and UAVs will attempt to cover all clusters and collect data from IoT nodes with the travelling route.

\section{Performance evaluation and discussion}

In this section, the authors will compare the proposed approach and the method of greedy in different scenarios. Authors use a travelled time used by FANET to end one round as a performance evaluation metric for our proposed method. Simulation environment and analytical results are presented in the following:

\subsection{Simulation environment}

In this environment, the area of size the $800 \times 800$ square meter network. Evaluation using Python software as a simulation platform, the PuLP (Python library for linear optimization) (Mitchell et al., 20111) has been implemented to perform the MILP optimization model on an OS system with a processor $2.3 \mathrm{GHz}$ QuadCore Intel Core i5 and 8 gigabytes of main memory. Table 3 shows the main parameters used in this simulation environment.

Table 3. Simulation Parameters

\begin{tabular}{|c|c|}
\hline Value & Simulation Parameter \\
\hline $800 \times 800 \mathrm{~m}^{2}$ & Area size \\
\hline 300 & Number of sensors \\
\hline 7101315 & Number of CHs \\
\hline 2346789 & Number of UAV \\
\hline $20 \mathrm{~m} / \mathrm{s}$ & Speeds of UAV \\
\hline $70 \mathrm{~m}$ & Heights of UAV \\
\hline $5070 \mathrm{~s}$ & Deadline Times $\tau$ \\
\hline $30 \mathrm{~s}$ & Sojourn Times $t_{i}$ \\
\hline $2000 \mathrm{bit}$ & Packet length \\
\hline $45 \mathrm{deg}$ & UAV Elevation Angles \\
\hline $200 \mathrm{kbps}$ & Data Transmission rate $f$ \\
\hline $200 \mathrm{~km}$ & $d_{\text {max }}$ \\
\hline $40 \mathrm{~m}$ & Transmission range \\
\hline $0.1 \mathrm{~J}$ & Initial energy $\left(E_{0}\right)$ (Alnuaimi et al.,2015) \\
\hline
\end{tabular}




\subsection{Results and discussion}

Authors evaluate the performance of the approach with a greedy approach that chooses the nearest IoT node for each UAV motion step to measure the efficiency of the proposed approach. A greedy algorithm is an algorithmic paradigm that follows the problem-solving heuristic of making the locally optimal choice at each stage to find an optimal global solution. (Curtis, 2003). Instead of contemplating the global situation, this algorithm chooses what appears to be the right thing to do at a given time (Gutin et al., 2002). The greedy method, in other words, is stuck in local minima and does not change itself based on UAV movements (Xu et al., 2016). Using the parameter value $u$, which is the output of the MILP model, this greedy approach initially considers the node from the cluster centres of the network as the initial position of the UAVs, and then each of the UAVs to cover the network and visit all the cluster centres in each step. After all the clusters are covered and the necessary data is obtained, UAV moves to the closest cluster. In the next step, in terms of energy consumption, the proposed approach and this greedy method are compared. Because energy consumption depends directly on the travelled time consumed by FANET, then authors use the UAV's travelled time as an energy comparison scale.

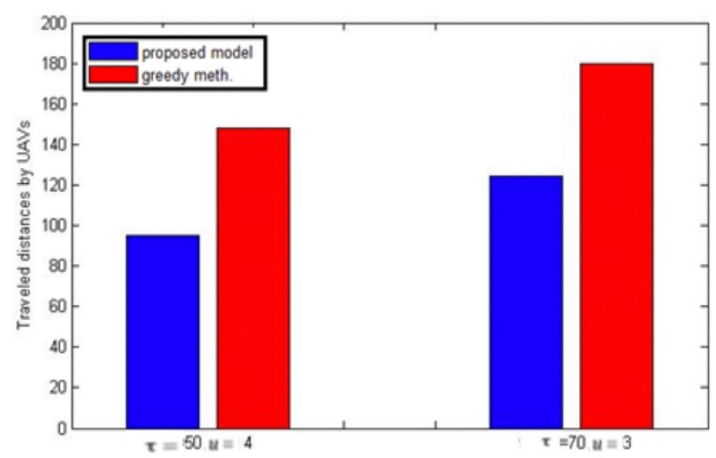

Fig. 2. UAV's travelled time comparison in the greedy approach and the proposed approach in $\tau=(50$ and 70) and $\alpha=0.9$ and $\beta=0.1$

Figure 2 shows the comparison of the UAV's travel time in the greedy approach and the proposed approach in $N=7$ at a different time deadline $\tau$. As stated in the description of the greedy method, the reason for the random selection of the starting nodes of the traversed path is compared to the proposed optimization model that attempts to select the appropriate position when selecting the source nodes to locate the beginning of the UAVs, a longer route goes through.

The effect of different values of the $\alpha$ and $\beta$ coefficients on the performance of the proposed model can be seen in the following figure, as defined in the selection section of the objective function in the same scenario. The comparison of UAV's traveled time of in the proposed approach and the greedy approach in the scenario with $N=10$ in different $t$ with a value of $\alpha=0.1, \beta=0.9$ is shown in Figure 3 .

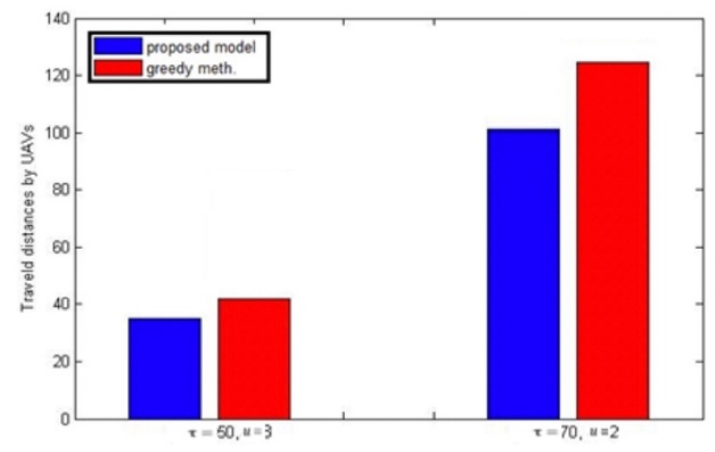

Fig. 3. UAV's travelled time comparison in the greedy approach and the proposed approach in $\tau=(50$ and 70) and $\alpha=0.1$ and $\beta=0.9$ 
The comparison of UAV's travelled time in the greedy approach and the approach method in $N=10$ in different $\tau$ with a value of $\alpha=0.9, \beta=0.1$ is shown in figure 4 .

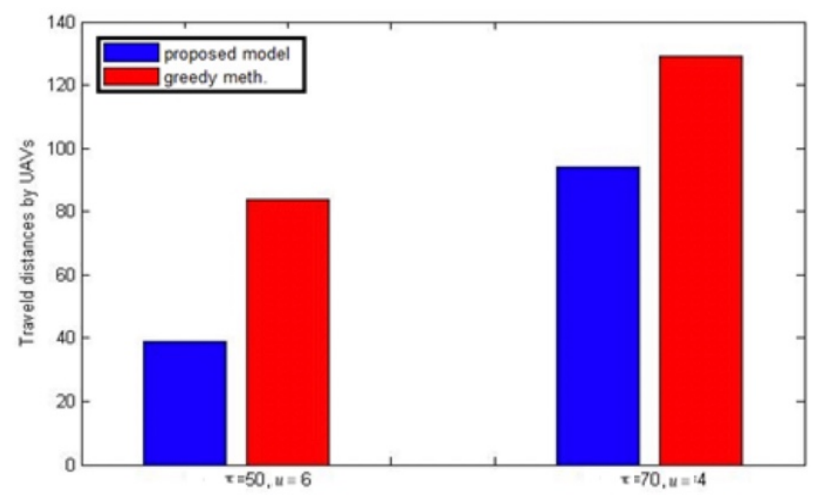

Fig. 4. UAV's travelled time comparison in the greedy approach and the proposed approach with $\tau=50.70$ and $\alpha=0.9$ and $\beta=0$.

The influence of choosing $\alpha$ and $\beta$ parameters on the performance of the proposed approach is presented in two deadline times $\tau=(50$ and 70$)$ and in the number of cluster heads $N=7,10$ is shown in the table below.

Table 4. UAV's travelled time comparison in the greedy approach and the proposed approach in $N=$

\begin{tabular}{|c|c|c|c|c|c|c|c|c|c|}
\hline \multicolumn{10}{|c|}{7,10 and $\tau=50,70$} \\
\hline$N$ & $\tau$ & $\alpha$ & $\beta$ & $U$ & $\boldsymbol{u}$ & $u_{c h}$ & $u_{t r j}$ & Traveled Time & Method \\
\hline \multirow{6}{*}{7} & \multirow{3}{*}{50} & 0.1 & 0.9 & 3 & 3 & 2 & 1 & 35 & Proposed model \\
\hline & & 0.9 & 0.1 & 3 & 3 & 2 & 1 & 35 & Proposed model \\
\hline & & - & - & - & 3 & 1 & 2 & 41 & Greedy method \\
\hline & \multirow{3}{*}{70} & 0.1 & 0.9 & 2 & 2 & 0 & 2 & 101 & Proposed model \\
\hline & & 0.9 & 0.1 & 2 & 2 & 0 & 2 & 101 & Proposed model \\
\hline & & - & - & - & 2 & 0 & 2 & 125 & Greedy method \\
\hline \multirow{8}{*}{10} & \multirow{4}{*}{50} & 0.1 & 0.9 & 6 & 4 & 1 & 3 & 95 & Proposed model \\
\hline & & 0.9 & 0.1 & 6 & 6 & 4 & 2 & 39 & Proposed model \\
\hline & & - & - & - & 4 & 0 & 4 & 148 & Greedy method \\
\hline & & - & - & - & 6 & 4 & 2 & 84 & Greedy method \\
\hline & \multirow{4}{*}{70} & 0.1 & 0.9 & 4 & 3 & 0 & 3 & 124 & Proposed model \\
\hline & & 0.9 & 0.1 & 4 & 4 & 1 & 3 & 94 & Proposed model \\
\hline & & - & - & - & 3 & 0 & 3 & 180 & Greedy method \\
\hline & & - & - & - & 4 & 0 & 4 & 129 & Greedy method \\
\hline
\end{tabular}

\section{Conclusion}

In this paper, the insufficient deadline time problem of data collection from a set of IoT targets using FANET is addressed. The problem is first formulated into a MILP model, and then the model is solved to find the minimum UAV travelled time concerning the deadline constraint for the critical level of the applications.

Simulation is performed in various scenarios to compare the efficiency of the proposed approach and the greedy approach. The simulation results showed that the proposed approach is capable of efficiently gathering data from IoT nodes to meet energy and deadline constraints as it relates to the crucial level of application. The simulation results also showed that the proposed approach can reduce the travel distance and travel time of FANET while guaranteeing the deadline time. 


\section{References}

Al-Turjman, F., \& Zahmatkesh, H. (2020). A Comprehensive Review on the Use of AI in UAV Communications: Enabling Technologies, Applications, and Challenges. In Unmanned Aerial Vehicles in Smart Cities (pp. 1-26). Springer, Cham.

Albu-Salih, A. T., \& Seno, S. A. H. (2018). Energy-efficient data gathering framework-based clustering via multiple UAV's in deadline-based WSN applications. IEEE Access, 6, 72275-72286.

ALBU-SALIH, A. T., \& SENO, S. A. H. (2018). Tour Time Minimization For Multiple UAV In Deadline Based WSN Applications. Journal of Theoretical \& Applied Information Technology, 96(17).

Alfattani, S., Jaafar, W., Yanikomeroglu, H., \& Yongacoglu, A. (2019, December). Multi-UAV Data Collection Framework for Wireless Sensor Networks. In 2019 IEEE Global Communications Conference (GLOBECOM) (pp. 1-6). IEEE.

Ali, Z. A., Masroor, S., \& Aamir, M. (2019). UAV based data gathering in wireless sensor networks. Wireless Personal Communications, 106(4), 1801-1811.

Alnuaimi, M., Shuaib, K., Alnuaimi, K., \& Abdel-Hafez, M. (2015). Data gathering in delay tolerant wireless sensor networks using a ferry. Sensors, 15(10), 25809-25830.

Bekmezci, I., Sahingoz, O. K., \& Temel, Ş. (2013). Flying ad-hoc networks (FANETs): A survey. Ad Hoc Networks, 11(3), 1254-1270.

Boursianis, A. D., Papadopoulou, M. S., Diamantoulakis, P., Liopa-Tsakalidi, A., Barouchas, P., Salahas, G., ... \& Goudos, S. K. (2020). Internet of Things (IoT) and Agricultural Unmanned Aerial Vehicles (UAVs) in Smart Farming: A Comprehensive Review. Internet of Things, 100187.

Caillouet, C., Giroire, F., \& Razafindralambo, T. (2019). Efficient data collection and tracking with flying drones. Ad Hoc Networks, 89, 35-46.

Curtis, S. A. (2003). The classification of greedy algorithms. Science of Computer Programming, 49(1-3), 125-157.

Dasgupta, R., \& Yoon, S. (2017). Energy-efficient deadline-aware data-gathering scheme using multiple mobile data collectors. Sensors, 17(4), 742.

Dong, M., Ota, K., Lin, M. et al. (2014). UAV-assisted data gathering in wireless sensor networks. J Supercomput 70, 1142-1155.

Gong, J., Chang, T. H., Shen, C., \& Chen, X. (2018). Flight time minimization of UAV for data collection over wireless sensor networks. IEEE Journal on Selected Areas in Communications, 36(9), 1942-1954.

Goudarzi, S., Kama, N., Anisi, M. H., Zeadally, S., \& Mumtaz, S. (2019). Data collection using unmanned aerial vehicles for Internet of Things platforms. Computers \& Electrical Engineering, 75, 1-15.

Gutin, G., Yeo, A., \& Zverovich, A. (2002). Travelling salesman should not be greedy: domination analysis of greedy-type heuristics for the TSP. Discrete Applied Mathematics, 117(1-3), 81-86.

Ho, D. T., Grotli, E. I., Sujit, P. B., Johansen, T. A., \& Sousa, J. B. (2015). Optimization of wireless sensor network and UAV data acquisition. Journal of Intelligent \& Robotic Systems, 78(1), 159-179. 
Khan, A. W., Abdullah, A. H., Anisi, M. H., \& Bangash, J. I. (2014). A comprehensive study of data collection schemes using mobile sinks in wireless sensor networks. Sensors, 14(2), 2510-2548.

Liu, B., \& Zhu, H. (2019). Energy-effective data gathering for UAV-aided Wireless Sensor Networks. Sensors, 19(11), 2506.

Liu, Y., Dai, H. N., Wang, Q., Shukla, M. K., \& Imran, M. (2020). Unmanned aerial vehicle for the internet of everything: Opportunities and challenges. Computer Communications.

Mitchell, S., OSullivan, M., \& Dunning, I. (2011). PuLP: a linear programming toolkit for python. The University of Auckland, Auckland, New Zealand.

Motlagh, N. H., Taleb, T., \& Arouk, O. (2016). Low-altitude unmanned aerial vehicles-based internet of things services: Comprehensive survey and future perspectives. IEEE Internet of Things Journal, 3(6), 899922.

Mozaffari, M., Saad, W., Bennis, M., \& Debbah, M. (2017). Mobile unmanned aerial vehicles (UAVs) for energy-efficient Internet of Things communications. IEEE Transactions on Wireless Communications, 16(11), 7574-7589.

Okcu, H., \& Soyturk, M. (2014). A distributed clustering approach for UAV integrated wireless sensor networks. International Journal of Ad Hoc and Ubiquitous Computing, 15(1-3), 106-120.

Otto, A., Agatz, N., Campbell, J., Golden, B., \& Pesch, E. (2018). Optimization approaches for civil applications of unmanned aerial vehicles (UAVs) or aerial drones: A survey. Networks, 72(4), 411-458.

Qin, Z., Li, A., Dong, C., Dai, H., \& Xu, Z. (2019). Completion Time Minimization for Multi-UAV Information Collection via Trajectory Planning. Sensors, 19(18), 4032.

Shakhatreh, H., Sawalmeh, A. H., Al-Fuqaha, A., Dou, Z., Almaita, E., Khalil, I., ... \& Guizani, M. (2019). Unmanned aerial vehicles (UAVs): A survey on civil applications and key research challenges. Ieee Access, 7, 48572-48634.

Sharma, V., \& Kumar, R. (2017). Cooperative frameworks and network models for flying ad hoc networks: a survey. Concurrency and computation: Practice and experience, 29(4), e3931.

Tuyishimire, E., Bagula, A., Rekhis, S., \& Boudriga, N. (2017, July). Cooperative data muling from ground sensors to base stations using UAVs. In 2017 IEEE Symposium on Computers and Communications (ISCC) (pp. 35-41). IEEE.

Xu, J., Solmaz, G., Rahmatizadeh, R., Turgut, D., \& Boloni, L. (2016). Internet of things applications: Animal monitoring with unmanned aerial vehicle. arXiv preprint arXiv:1610.05287.

Zhan, C., \& Zeng, Y. (2019). Completion time minimization for multi-UAV-enabled data collection. IEEE Transactions on Wireless Communications, 18(10), 4859-4872.

Submitted: $\quad 01 / 10 / 2020$

Revised: $\quad 01 / 12 / 2020$

Accepted: $\quad 11 / 03 / 2021$

DOI: $\quad 10.48129 /$ kjs.v49i1.10654 